\title{
ИНЖЕНЕРНАЯ ГЕОЛОГИЯ
}

УДК 624.131

\section{Инженерно-геологическое районирование, основанное на многомерной оценке классификационного показателя}

\section{В.В. Середин, П.А. Красильников}

Пермский государственный национальный исследовательский университет, 614000, Пермь, ул. Букирева, 15. E-mail: kafedra.ingeo@gmail.com (Статья поступила в редакциию 22 февраля 2016 г.)

Приводятся результаты разработки методики инженерно-геологического районирования на основе многомерной оценки классификационного показателя. В практике районирования существуют, по крайней мере, три группы способов оценки геологических признаков безразмерной величиной: балльный, нормирования и вероятностно-статистический. По мнению авторов, при разработке методов районирования наиболее перспективна статистическая оценка геологических признаков и классификационного показателя, которая позволяет минимизировать субъективизм на этапах выбора классификационного показателя и обоснования границ таксонов. Поэтому целью исследования является разработка методики районирования территории на основе многомерной статистической оценки классификационного показателя. Процедура районирования сводится к выбору классификационного показателя (Кр), оценке его многомерным статистическим критерием, обоснованию граничных значений, составлению модели районирования и выделению таксонов. Граничные значения классификационного показателя Rо вычисляются по данным дискриминантной функции. Проведена апробация предложенной методики, составлена схема районирования.

Ключевые слова: инженерно-геологическое районирование, классификационный показатель, дискриминантный анализ, статистическая оценка.

DOI: 10.17072/psu.geol.31.48

\section{Актуальность}

Актуальность работы обусловлена тем, что зачастую на этапе обоснования инвестиций строительства крупных объектов, к примеру нефтепровода ВСТО (Восточная Сибирь-Тихий океан) требуется проведение инженерно-геологического районирования территории строительства.

Анализ результатов районирования, полученных разными методами, показал, что они не всегда сопоставимы между собой. Это обусловлено рядом причин, в том числе:

- способами перевода геологических признаков из размерных в безразмерные величины;

- технологией выбора и оценки обобщенного показателя, выступающего в качестве классификационного признака при районировании.

(C) Середин В.В., Красильников П.А., 2016 
В практике районирования существуют, по крайней мере, три группы способов оценки геологических признаков безразмерной величиной: балльный [3, 6, 13], нормирования $[1,8]$ и вероятностностатистический $[12,14]$. Наиболее перспективна статистическая оценка геологических признаков и классификационного показателя. Она позволяет минимизировать субъективизм на этапах выбора классификационного показателя и обоснования границ таксонов.

Поэтому целью исследования является разработка методики районирования территории на основе многомерной статистической оценки классификационного показателя.

Процедура районирования сводится к выбору классификационного показателя (Кр), оценке его многомерным статистическим критерием, обоснованию граничных значений Кр, составлению модели районирования и выделению таксонов.

\section{Выбор и оценка классификационного показателя (Кр)}

Для решения поставленной задачи целесообразно использовать дискриминантный анализ, сущность которого сводится к следующему. Геологические показатели, например, мощность пласта, переводятся в безразмерные величины. Для повышения надежности используют не один, а несколько геологических показателей. В качестве безразмерного значения геологических показателей может выступать значение $\mathrm{R}$ дискриминантной функции, которое в дальнейшем используется как классификационный показатель $\mathrm{R}=\mathrm{K}_{\mathrm{p}}$.

Для вычисления R следует использовать линейную дискриминантную функцию (ЛДФ), при этом для расчета ЛДФ необходима геологическая информация по эталонным участкам исследуемой территории, например, участкам, где сооружения находятся в устойчивом (G-1) и неустойчивом (G-2) состоянии.
Процедура расчета $\mathrm{R}$ сводится к следующему. Если обозначить через $\mathrm{X}_{\mathrm{ij}}$ значение переменной с номером i в точке наблюдения с номером ј, взятой из первой выборки (G-1), то в результате может быть получена матрица $\mathrm{W}_{1}$ порядка $\mathrm{m}$ и $\mathrm{n}_{1}$ peзультатов наблюдений над этой выборкой:

$$
W_{1}=\left[\begin{array}{cccc}
X_{11} & X_{12} & \cdot & X_{1 n_{1}} \\
X_{21} & X_{22} & \cdot & X_{2 n_{1}} \\
\cdot & \cdot & \cdot & \cdot \\
X_{m 1} & X_{m 2} & \cdot & X_{m n_{1}}
\end{array}\right] \quad \text {. }
$$

Обозначим через $\mathrm{X}^{1}{ }_{\text {ij }}$ результат измерения переменной с номером і в точке с номером j, взятой из второй выборки (G-2). $\mathrm{B}$ результате получим матрицу $\mathrm{W}_{2}$ порядка $\mathrm{m} \times \mathrm{n}_{2}$ :

$W_{2}=\left[\begin{array}{cccc}X_{11}^{1} & X_{12}^{1} & \cdot & X_{1 n_{2}} \\ X_{21}^{1} & X_{22}^{1} & \cdot & X_{2 n_{2}}^{1} \\ \cdot & \cdot & \cdot & \cdot \\ X_{m 1}^{1} & X_{m 2}^{1} & \cdot & X_{m n_{2}}^{1}\end{array}\right]$,

где $\mathrm{m}$ - число геологических показателей;

$\mathrm{n}_{1}, \mathrm{n}_{2}$ - объемы первой и второй выборок.

Для построения ЛДФ составляются матрицы центрированных сумм квадратов и смещенных произведений $\widetilde{S}_{W 1}$ и $\widetilde{S}_{W 2}$, по ним вычисляется выборочная матрица

$$
M=\frac{\widetilde{S}_{W 1}+\widetilde{S}_{W 2}}{n_{1}+n_{2}-2} .
$$

Для определения коэффициентов линейной дискриминантной функции находится обратная выборочная ковариационная матрица - матрица С. Коэффициенты дискриминантной функции вычисляют по формуле

$$
a_{m}=\sum_{i=1}^{m} C_{m j}\left(\bar{X}_{m}^{W 1}-\bar{X}_{m}^{W 2}\right),
$$

где $\mathrm{C}_{\mathrm{mj}}$ - элементы обратной матрицы $\mathrm{C}$, $\bar{X}$ - среднее значение соответствующего показателя.

После чего рассчитывается линейная дискриминантная функция 
$\mathrm{R}=\mathrm{a}_{\mathrm{m} 1} \cdot \mathrm{m}_{1}+\mathrm{a}_{\mathrm{m} 2} \cdot \mathrm{m}_{2}+\mathrm{a}_{\mathrm{m} 3} \cdot \mathrm{m}_{3}+\mathrm{a}_{\mathrm{mn}} \cdot \mathrm{m}_{\mathrm{n}}+\mathrm{b}$,

где $\mathrm{m}_{1}, \mathrm{~m}_{2}, \mathrm{~m}_{3}, \mathrm{~m}_{\mathrm{n}}$ - значения геологических показателей в каждой точке опробования, $\mathrm{b}$ - свободный член.

\section{Определение граничных значений}

Одной из основных задач дискриминантного анализа является установление поверхности разделения между исследуемыми подмножествами, в нашем случае между таксонами $\left(\mathrm{R}_{\mathrm{o}}\right)$. При этом чем больше геологических признаков будут участвовать в этом расчете, тем надежнее будет установлена граница между таксонами. Следует отметить, что наиболее эффективно этот метод (дискриминантный) работает в том случае, когда между двумя классами не наблюдается четко выраженной границы, т. е. поле рассеивания одного класса накладывается на поле рассеивания другого класса. Для расчета $\mathrm{R}_{\mathrm{o}}$ следует использовать следующую зависимость:

$$
R_{0}=\frac{1}{2} \sum_{i=1}^{m} a_{m}\left(\bar{X}_{m}^{W 1}-\bar{X}_{m}^{W 2}\right),
$$

при $\mathrm{R}>\mathrm{R}_{\mathrm{o}}$ объект принадлежит к классу G1 , а при $\mathrm{R}<\mathrm{R}_{\text {о }}$ к классу $\mathrm{G}-2$.

Надежность классификации определяют с помощью критериев Пирсона и Фишера.

Таблица 1. Модель районирования

\begin{tabular}{clc}
\hline Таксон & $\begin{array}{c}\text { Состояние объек- } \\
\text { та }\end{array}$ & $\begin{array}{c}\text { Значение } \\
\mathrm{K}_{\mathrm{p}}=R\end{array}$ \\
\hline \hline $\mathrm{G}-1$ & Устойчивое $^{\text {Heутойчиое }}$ & $R>R_{\mathrm{o}}$ \\
$\mathrm{G}-2$ & Неустой & $R<R_{\mathrm{o}}$ \\
\hline
\end{tabular}

Таксоны выделяем следующим образом: исследуемая территория разделяется на подобласти (с равными площадями), каждая из которых характеризуется численными значениями классификационного показателя R. После чего выделяются и описываются таксоны по методике [9].

\section{Пример практической реализации}

Между пунктами А и В построен и эксплуатируется в течение 35 лет нефтепровод. Его протяженность составляет 20,4 км. Анализ состояния трубопровода показал, что на пикетах (ПК) с 19 по 35 он находится в устойчивом состоянии G-1 (аварии не зафиксированы), а на ПК 1-11, наоборот, в неудовлетворительном состоянии G-2, о чем свидетельствуют ежегодные аварийные ситуации.

Формулируется задача: оценить состояние трубопровода по данным изысканий. Результаты представить в виде карты районирования. Исходные данные: трубопровод диаметром 89 мм, материал Ст-35, глубина заложения 2,8 м.

Инженерно-геологическое изучение территории включало в себя бурение скважин, описание керна, отбор проб и монолитов по пикетам (ПК). Нумерация ПК начиналась с пункта А.

В геологическом строении принимают участие (сверху вниз): песок средней крупности мощностью до 8,9 м, глина мягкопластичная мощностью до 5,3 м, суглинок - мягкопластичный мощностью до 5,3 м и песчаник крепкий, трещиноватый, мощностью (вскрытой) до 5 м. Для реализации поставленной задачи целесообразно использовать программу «Статистика», которая широко применяется для обработки инженерно-геологической информации.

\section{Алгоритм выполнения работ}

1. В расчетах участвуют следующие геологические показатели: абсолютная отметка земной поверхности $\left(\mathrm{AO}_{3 . \Pi}\right)$, мощность песка $\left(\mathrm{m}_{\Pi}\right)$, мощность глины $\left(\mathrm{m}_{\text {г }}\right)$, мощность суглинка $\left(\mathrm{m}_{\text {суг }}\right)$ и абсолютная отметка кровли песчаника $\left(\mathrm{AO}_{\text {п }}\right)$.

2. В таблице исходных данных выделяем по пикетам эталонные участки (группы G-1 и G-2).

3. Вычисляем среднее, стандартное отклонения и мах и міn по каждому показателю. Результаты расчетов представлены в табл. 2. 
Таблица 2. Описательные статистики (исходные данные районирования)

\begin{tabular}{llllll}
\hline \hline & N набл. & Среднее & Минимум & Максимум & $\begin{array}{l}\text { Стандартное } \\
\text { отклонение }\end{array}$ \\
\hline $\begin{array}{l}\text { Абсолютная отметка } \\
\text { земной поверхности, м }\end{array}$ & 51 & 101,14 & 99,6 & 102,8 & 1,034 \\
Мощность песка, м & 51 & 4,80 & 0,6 & 8,9 & 2,6584 \\
Мощность глины, м & 51 & 3,26 & 0,7 & 5,3 & 1,525 \\
Мощность суглинка, м & 51 & 3,62 & 1,9 & 5,3 & 1,101 \\
$\begin{array}{l}\text { Абсолютная отметка } \\
\text { кровли песчаника, м }\end{array}$ & 51 & 89,42 & 86,9 & 92,2 & 1,767 \\
\hline
\end{tabular}

4. Производим оценку надежности выделения эталонных участков G-1 и G-2 по критерию «Хи-квадрат». Расчетное значение составляет $\chi_{\mathrm{p}}^{2}=111,45$. Затем вычисляем табличное значение $\chi_{\mathrm{r}}^{2}=9,5$ при уровне значимости $\alpha=0,05$ и степенях свободы $\mathrm{K}=\Gamma-3=7-3=4$, где $\Gamma-$ число интервалов. Отсюда $\chi_{\mathrm{p}}^{2}=111,45>\quad \chi_{\mathrm{r}}^{2}=9,5$, это свидетельствует о том, что эталонные участки G-1 и G-2 различаются между собой.

5. Рассчитываем коэффициенты дискриминантной функции (табл. 3).

Таблица 3. Исходные коэффициенты для канонических переменных

\begin{tabular}{|l|c|}
\hline $\begin{array}{l}\text { Абс. отм. зем.поверхн. }\left(\mathrm{AO}_{\text {з.п. }}\right), \\
\text { м }\end{array}$ & 10,732 \\
\hline Мощность песка $\left(\mathrm{m}_{\text {п }}\right), \mathrm{m}$ & $-0,868$ \\
\hline Мощность глины $\left(\mathrm{m}_{\text {г }}\right), \mathrm{m}$ & $-1,942$ \\
\hline Мощность суглинка $\left(\mathrm{m}_{\text {суг }}\right), \mathrm{m}$ & $-0,101$ \\
\hline $\begin{array}{l}\text { Абс. отм. кровли песчаника } \\
(\text { АО }\end{array}$ & $-1,397$ \\
\hline Константа & $-946,058$ \\
\hline Собственные значения & 113,755 \\
\hline Кумулятивная доля & 1,000 \\
\hline
\end{tabular}

Используя эти коэффициенты (табл.3), рассчитываем дискриминантную функцию, которая имеет следующий вид:

$\mathrm{R}=10,734 \cdot \mathrm{AO}_{3 . \Pi}-0,868 \cdot \mathrm{m}_{\Pi}-1,942 \cdot \mathrm{m}_{\Gamma}$ $0,101 \cdot \mathrm{m}_{\text {суг }}-1,397 \cdot \mathrm{AO}_{\text {п }}-946,058$.

5. Ррассчитываем значения $\mathrm{R}$ для каждой точки наблюдения (табл. 4).

Граничное значение дискриминантной функции $\mathrm{R}_{\mathrm{o}}$, которое делит наблюдения на классы G-1 и G-2, определяется следующим образом: в дискриминантную функ- цию $\mathrm{R}(\mathrm{Z})=10,734 \cdot \mathrm{AO}_{3 . \Pi} \quad-0,868 \cdot \mathrm{m}_{\text {п }} \quad$ $1,942 \cdot \mathrm{m}_{\Gamma}-0,101 \cdot \mathrm{m}_{\mathrm{cyг}}-1,397 \cdot \mathrm{AO}_{\text {п }}-946,058$ подставляем средние значения геологических признаков (табл.2) и рассчитываем $R_{\mathrm{o}}=1085,6077-4,1646-6,34-0,3659$ -

$124,9219-946,058=3,7573$.

При $R>R_{0}=3,7573$ объект принадлежит к классу G-2 и при $R<R_{\mathrm{0}}=3,7573$ объект принадлежит к классу G-1.

Модель районирования составляется по данным $\mathrm{R}_{\text {o }}$ (табл. 5).

Зная численные значения $\mathrm{K}_{\mathrm{p}}=R$ в каждой точки наблюдения (табл.4), используя модель районирования (табл.5), типизируем трассу нефтепровода. Выделяются два таксона:

таксон 1 представлен участком «ПК19 -ПК51». На этом участке в основании трубопровода залегает песок средней крупности. Значения классификационного показателя составляют $\mathrm{R}=\mathrm{K}_{\mathrm{p}}>3,7573$;

таксон 2 представлен участком трассы трубопровода «ПК1 - ПК18». На этом участке в основании трубопровода залегает мягкопластичная глина. Значения классификационного показателя $\mathrm{R}=\mathrm{K}_{\mathrm{p}}<3,7573$.

\section{Выводы}

1. Разработана методика инженерногеологического районирования территорий на основе многомерного статистического классификационного показателя, в качестве которого предлагается использовать критерий $\mathrm{R}$ дискриминантной функции. 
Таблица 4. Значение классификационного показателя $(R)$ на разных участках трассы

\begin{tabular}{cccccccccc}
\hline $\begin{array}{c}\text { № пи- } \\
\text { кета }\end{array}$ & $\mathrm{R}$ & $\begin{array}{c}\text { № пи- } \\
\text { кета }\end{array}$ & $\mathrm{R}$ & $\begin{array}{c}\text { № пике- } \\
\text { та }\end{array}$ & $\mathrm{R}$ & $\begin{array}{c}\text { № пи- } \\
\text { кета }\end{array}$ & $\mathrm{R}$ & $\begin{array}{c}\text { № пи- } \\
\text { кета }\end{array}$ & $\mathrm{R}$ \\
\hline 1 & $-12,80$ & 11 & $-11,97$ & 21 & 7,71 & 31 & 9,00 & 41 & 11,29 \\
2 & $-13,62$ & 12 & $-9,35$ & 22 & 8,85 & 32 & 8,14 & 42 & 11,80 \\
3 & $-12,36$ & 13 & $-8,84$ & 23 & 10,40 & 33 & 8,87 & 43 & 12,89 \\
4 & $-13,32$ & 14 & $-6,91$ & 24 & 9,33 & 34 & 7,87 & 44 & 13,36 \\
5 & $-14,33$ & 15 & $-3,41$ & 25 & 8,46 & 35 & 8,25 & 45 & 13,60 \\
6 & $-12,23$ & 16 & $-2,52$ & 26 & 8,40 & 36 & 7,89 & 46 & 15,02 \\
7 & $-13,09$ & 17 & 0,43 & 27 & 8,62 & 37 & 8,43 & 47 & 16,06 \\
8 & $-12,71$ & 18 & 2,79 & 28 & 7,16 & 38 & 10,04 & 48 & 16,93 \\
9 & $-12,31$ & 19 & 4,90 & 29 & 8,27 & 39 & 10,38 & 49 & 17,22 \\
10 & $-11,81$ & 20 & 8,02 & 30 & 8,31 & 40 & 10,61 & 50 & 16,05 \\
\hline
\end{tabular}

Таблица 5. Модель районирования

\begin{tabular}{cll}
\hline Таксон & Состояние объекта & Значение $\mathrm{K}_{\mathrm{p}}=R$ \\
\hline $\mathrm{G}-1$ & Устойчивое & $R>R_{\mathrm{o}}=3,7573$ \\
$\mathrm{G}-2$ & Неустойчивое & $R<R_{\mathrm{o}}=3,7573$ \\
\hline
\end{tabular}

2. Граничные значения классификационного показателя $\mathrm{R}_{\text {o }}$ вычисляются по данным дискриминантной функции.

3. Проведена апробация предложенной методики, составлена схема районирования.

\section{Библиографический список}

1. Бондарик Г.К., Пендин В.В. Методика количественной оценки инженерногеологических условий и специального инженерно-геологического районирования // Инженерная геология. 1982. № 4. С. $82-$ 89.

2. Копылов И.С., Коноплев А.В., Голдырев В.В., Кустов И.В., Красильников П.А. К вопросу об обеспечении геологической безопасности развития городов // Фундаментальные исследования. 2014. № 9-2. С. 355359.

3. Красильников П.А., Коноплев А.В., Хронусов В.В., Барский М.Г. Геоинформационное обеспечение экономической оценки природно-ресурсного потенциала территорий Пермского края // Экономика региона. 2009. № 1. C. 143-151.

4. Красильников П.А., Середин В.В., Леонович $M . Ф$. Исследование распределения углеводородов по разрезу грунтового массива // Фундаментальные исследования. 2015. № 2-14. C. 3100-3104.
5. Круподеров В.С., Трофимов В.Т., Чекрыгина, С.Н. Научно-методические подходы и принципы составления современной инженерно-геологической карты России масштаба 1:2500000 // Разведка и охрана недр. 2008. № 6. С. 24-26.

6. Минц А.А. Вопросы комплексной экономической оценки природных условий и естественных ресурсов в свете задач современной географии // Изв. АН СССР. Сер. География. 1965.№2.

7. Новопоселенских Л.А., Середин В.В. Экономическое состояние инженерноизыскательской отрасли. Геология и полезные ископаемые Западного Урала. 2015. № 15.C.168-171.

8. Оздоева Л.И. Использование интегрального показателя инженерно-геологических условий при крупномасштабном инженерно-геологическом районировании городских территорий // Изв. вузов. Геология и разведка.1981. №8.С.70-74.

9. Пендин В.В., Ганова С.Д. Геоэкологический мониторинг территорий расположения объектов транспорта газа в криолитозоне ПНИИИС. М., 2009. 236 с.

10. Пушкарева М.В., Середин В.В., Лейбович Л.О., Чиркова А.А., Бахарев А.О. Инженерно-экологическая оценка территорий запасов подземных вод в связи с разработкой нефтяных месторождений // Защита окружающей среды в нефтегазовом комплексе. 2013. № 2. C. 9-13. 
11. Середин В.В., Галкин В.И., Растегаев А.В., Лейбович Л.О., Пушкарева М.В. Прогнозирование карстовой опасности при инженерно-геологическом районировании территорий // Инженерная геология. 2012. № 2. С. 40-45.

12. Середин В.В., Галкин В.И., Пушкарева М.В., Лейбович Л.О., Сметанин С.Н. Вероятностно-статистическая оценка инженерно-геологических условий для специального районирования // Там же. 2011. № 4. С. 42-47.

13. Середин В.В., Пушкарева М.В., Лейбович Л.О., Бахарева Н.С. Методика инженерно- геологического районирования на основе балльной оценки классификационного признака // Там же. № 3. С. 20-25.

14. Толмачев B.B. Вероятностный подход при оценке устойчивости закарстованных территорий и проектировании противокарстовых мероприятий // Там же. 1980. № 3. С. 98-107.

15. Трофимов B.T. Теоретические вопросы инженерно-геологического районирования // Вестник Московского университета. Сер. Геология. 1979. № 1. С. 94-106.

\title{
Methodology of Geological Engineering Zonation Based on a Multidimensional Assessment of Classification Index
}

\author{
V.V. Seredin, P.A. Krasilnikov \\ Perm State University, 15 Bukireva Str., Perm 614990, Russia \\ E-mail: kafedra.ingeo@gmail.com
}

The article presents the results of development of the geoengineering zonation method based on multidimensional assessment of the classification index. At least three groups of assessment of geological indicators by dimensionless quantity are used in the practice of geoengineering zonation: a weighted scoring method, rating method, and statistical method. Authors consider the multidimensional estimation of geological indicators and classification index as the most effective technique for geological engineering zonation. Usage of this methodology allows minimizing a researcher subjectivity in selection of the classification parameter and taxon's boundaries reasoning. Zonation procedure includes a selection of classification indicator, evaluation of multidimensional statistical criterion, reasoning of the boundaries conditions, creation of the zonation model, and taxons definition. Boundary values of classification indicator are calculated using a discriminant function. The zonation scheme of the oil pipeline area was made using developed methodology.

Key words: geological engineering zonation, classification indicator, discriminant analysis, statistical estimation.

\section{References}

1. Bondarik G.K., Pendin V.V. 1982. Metodika kolichestvennoy otsenki inzhenernogeologicheskikh usloviy i spetsialnogo inzhenerno-geologicheskogo rayonirovaniya [Methodology of quantitative estimation of geological engineering conditions and special geoengineering zonation]. Inzhenernaya geologiya. 4: 82-89. (in Russian)
2. Kopylov I.S., Konoplev A.V., Goldyrev V.V., Kustov I.V., Krasilnikov P.A. 2014. K voprosu ob obespechenii geologicheskoy bezopasnosti razvitiya gorodov [On the problem of providing the geological safety of urban development]. Fundamentalnye issledovaniya. 9-2: 355-359. (in Russian)

3. Krasilnikov P.A., Konoplev A.V., Hronusov V.V., Barskiy M.G. 2009. Geoinformatsionnoe obespechenie ekonomicheskoy otsenki prirodno-resursnogo potentsiala territoriy 
Permskogo kraya [Geoinformation support of the economic assessment of natural resources potential of the Perm kray territory]. Ekonomika regiona. 1: 143-151. (in Russian)

4. Krasilnikov P.A., Seredin V.V., Leonovich M.F. 2015. Issledovanie raspredeleniya uglevodorodov po razrezu gruntovogo massiva [Study of hydrocarbons distribution within the cross-section of a soil massif]. Fundamentalnye issledovaniya. 2-14: 3100-3104. (in Russian)

5. Krupoderov V.S., Trofimov V.T., Chekrygina S.N. 2008. Nauchno-metodicheskie podkhody i printsipy sostavleniya sovremennoy inzhenerno-geologicheskoy karty Rossii masshtaba 1:2500000 [Scientific and methodical approach and principals of compilation of modern geological engineering map of Russia of 1:2500000 scale]. Razvedka i okhrana nedr. 6: 24-26. (in Russian)

6. Mints A.A. 1995. Voprosy kompleksnoy ekonomicheskoy otsenki prirodnykh usloviy i estestvennykh resursov $\mathrm{v}$ svete zadach sovremennoy geografii [Problems of integrated economic assessment of natural conditions and resources according the demands of modern geography]. Izv. AN SSSR. Ser. Geograf. 2: 65-76. (in Russian)

7. Novoposelenskikh L.A., Seredin V.V. 2015. Ekonomicheskoe sostoyanie inzhenernoizyskatelskoy otrasli [Economic state of the engineering surveying]. Proc. of Conf. Geologiya i poleznye iskopaemye Zapadnogo Urala. 15:168-171. (in Russian)

8. Ozdoeva L.I. 1981. Ispolzovanie integralnogo pokazatelya inzhenerno-geologicheskikh usloviy pri krupnomasshtabnom inzhenernogeologicheskom rayonirovanii gorodskikh territoriy [Usage of the integral parameter of geological engineering conditions for the detailed scale zoning of urban territories]. Izv. Vuzov. Geologija i razvedka. 8: 70-74. (in Russian)

9. Pendin V.V., Ganova S.D. 2009. Geoekologicheskiy monitoring territoriy raspolozheniya obyektov transporta gaza $\mathrm{v}$ kriolitozone [Geoecological monitoring of territories of gas transportation facilities in the permafrost zone]. PNIIIS, Moskva, p. 236. (in Russian)
10. Pushkareva M.V., Seredin V.V., Leybovich L.O., Chirkova A.A., Bakharev A.O. 2013. Inzhenerno-ekologicheskaya otsenka territoriy zapasov podzemnykh vod v svyazi s razrabotkoy neftyanykh mestorozhdeniy [Engineering ecological assessment of territories of the groundwater resources relative to the oil fields development]. Zashchita okruzhayushchey sredy v neftegazovom komplekse. 2: 9-13. (in Russian)

11. Seredin V.V., Galkin V.I., Rastegaev A.V., Leybovich L.O., Pushkareva M.V. 2012. Prognozirovanie karstovoy opasnosti pri inzhenerno-geologicheskom rayonirovanii territoriy [Karst emergency prediction for the geoengineering zonation of territories]. Inzhenernaya geologiya. 2: 40-45. (in Russian)

12. Seredin V.V., Galkin V.I., Pushkareva M.V., Lejbovich L.O., Smetanin S.N. 2011. Veroyatnostno-statisticheskaya otsenka inzhenerno-geologicheskikh usloviy dlya spetsialnogo rayonirovaniya [Statistical estimation of the geoengineering conditions for special zonation]. Inzhenernaya geologiya. 4: 42-47. (in Russian)

13. Seredin V.V., Pushkareva M.V., Leybovich L.O., Bakhareva N.S. 2011. Metodika inzhenerno-geologicheskogo rayonirovaniya na osnove balnoy otsenki klassifikatsionnogo priznaka [Methodology of geoengineering zonation based on the weighted scoring estimation of classification index]. Inzhenernaya geologiya. 3: 20-25. (in Russian)

14. Tolmachev V.V. 1980. Veroyatnostnyy podkhod pri otsenke ustoychivosti zakarstovannykh territoriy i proektirovanii protivokarstovykh meropriyatiy [Probabilistic approach for the assessment of stability of the karstified areas and karst hazard mitigation planning]. Inzhenernaya geologiya. 3: 98107. (in Russian)

15. Trofimov V.T. 1979. Teoreticheskie voprosy inzhenerno-geologicheskogo rayonirovaniya [Theoretical problems of geoengineering zonation]. Vestnik Moskovskogo universiteta. Serija Geologija. 1: 94-106. (in Russian) 